\title{
Enhancing interoperability in the design process, the PROSIT approach
}

\author{
Umberto Cugini ${ }^{1}$, Gaetano Cascini ${ }^{2}$, Marco Ugolotti ${ }^{1}$ \\ Politecnico di Milano, Dipartimento di Meccanica, Milano, Italy \\ \{umberto.cugini, marco.ugolotti\}@polimi.it \\ 2 Università di Firenze, Dip. di Meccanica e Tecnlogie Ind.li, \\ Florence, Italy, gaetano.cascini@unifi.it
}

\begin{abstract}
The paper presents a methodology developed within the PROSIT project, aimed at the improvement of the product development cycle through the integration of Computer-Aided Innovation systems with Optimization and PLM/EKM systems. The interoperability of these tools is obtained through the adoption of Optimization systems as design analysis means and the definition of formalized and validated procedures and guidelines. The logic of the proposed methodology is explained through a detailed study case related to the design of a plastic wheel for light moto-scooters.
\end{abstract}

\section{Introduction}

The necessity to succeed in the global market or, at least, to survive in such a competitive environment, forces industries to systematically innovate products, to reduce their costs and to introduce them faster.

The achievement of this goal has led to the introduction of new technologies and new working approaches in the product development domain in order to improve (often in a very radical way) design activity performances. In the conceptual design phase, for instance, several methods and tools have been developed over the years to support the systematic transfer of innovative solutions among different technical fields. It is the case of Computer Aided Innovation (CAI) systems, which help engineers and technicians addressing design problems and guiding them to new possible solving approaches [1]. Moreover, methods for structural and topological optimization (TO), based on the use of a generative algorithm, are actually used by a lot of practitioners to obtain optimal geometrical solutions [2]. Knowledge-Based Engineering systems (KBE) finally, support designers' activity through rules and knowledge re-use, thus reducing the product development time without effecting its functionality, quality and security [3]. These tools have demonstrated their relevant potentialities to increase the effectiveness of specific design activities where they are

Please use the following format when citing this chapter:

Cugini, U., Cascini, G., Ugolotti, M., 2007, in IFIP International Federation for Information Processing, Volume 250, Trends in Computer Aided Innovation, ed. León-Rovira, N., (Boston: Springer), pp. 189-199. 
used. Technological potentials of these systems, however, especially the potential od a smooth and effective integration, have not been fully exploited and it is mainly due, on one side, to a poor integration between different applications, and on the other side by the difficulty to fully understand how to take advantages of these tools and how to effectively use them in the context of the product development process. Indeed, it has been estimated that the United States industry spends billions of dollars as a result of poor interoperability between computer-aided engineering software tools [4].

A full integration of these technologies is still far to be reached [5], and a big effort is required to set up successful collaborations and to push companies to focus their attention on the adoption of new organizational paradigms to better coordinate the design activity in such a context. The capability to support in a more integrated way all the stages of the product development process will be one of the most important competitive factors for these systems in the next years. In a concurrent engineering view, in fact, it is required that the phases of conceptual design, optimization and detailed design would be integrated as far as possible (Fig. 1). In order to reduce development time and increase activities' effectiveness, design and product development process have to be considered as a continuous iteration among these phases.

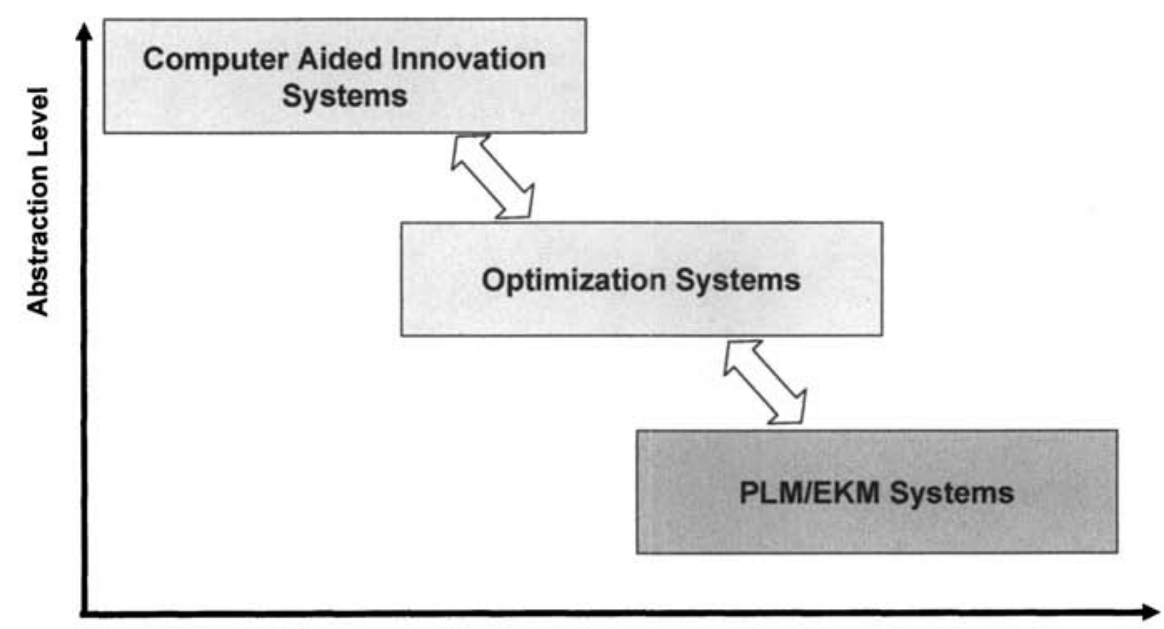

Product development

Fig. 1. Methods and systems to support product development process

Real potentialities of CAI, TO and KBE tools are still far to be reached. Lack of integration between different systems has reduced the impact of these tools in the design process. One of the main reasons is that these applications have been developed in the past mainly as standalone solutions, as islands of automation, unable in most cases to communicate and interoperate each other. The development of a more interoperable design process, in fact, is not only a matter of new advanced and independent IT solutions, but it has to be deeply focalized in the development of 
methodologies and guidelines aiming at supporting design teams in a more effective and efficient way, improving the advantages provided by the adoption of available product development systems, data management activities and knowledge sharing among design teams.

In the last years several authors have tried to integrate optimization techniques in product design processes with classical approaches such as feature-recognition [6] and design-by-feature [7]. Innovative frameworks have been also proposed to introduce optimization processes from the conceptual design stage, for instance by using hybrid CAD/FEM models built up by predefined components with predefined structural characteristics [8]. Other attempts have been tested, aimed at integrating optimization and other design activities through iterative approaches where the initial geometry is submitted to a Geometry (CAD) $\rightarrow$ Simulation (FEM) $>>$ Optimization $>$ Geometry (CAD) cycle [9]. However the proposed solutions implemented so far did not result in an integrated approach, neither have been expressed into formalized procedures for the systematic introduction of structural optimization systems in the design process.

Finally, the introduction of innovations into a process requires a preliminary study to estimate the impact of the proposed changes, before making the required investment to introduce them. It is thus necessary to adopt a systematic approach to the definition of a process modification through an effective and efficient introduction of technologies, and to support new technologies evaluation and impact introduction analysis, considering aspects as costs, times, design errors and iteration reduction, etc. The authors have already developed in the past some evaluation metrics for the assessment of the benefits provided by the adoption of new technologies in a product development process as a comparison between its As-Is and To-Be models [10].

This paper tries to put in evidence the meaning of interoperability in the design process and to present a methodology developed by the authors for the design and implementation of more interoperable design environments. The methodology here presented has been developed in the frame of an Italian ministry co-financed project PRIN 2005, named PROSIT (From Systematic Innovation to Integrated Product Development).

The main objective of this project is to study and test possible solutions for integrating innovative tools such as PLM (Product Lifecycle Management) and EKM (Engineering Knowledge Management), with CAI systems and TO tools within the product development cycle. The rationale of the proposed research is the lack of formalized and validated procedures allowing the systematic introduction and integration of these tools in the design process.

\section{Methodology}

According to the diagram of Fig. 1, the PROSIT project aims at bridging three different classes of product development methods and systems, CAI and Optimization systems from one side, Optimization systems and PLM/EKM tools to the other. 
The main idea of the methodology developed in the frame of the project to link CAI and Optimization systems is the adoption of the latter tools not just to generate optimized solutions, but also as a design analysis tool, capable to outline critical aspects of a mechanical component in terms of conflicting design requirements or parameters.

The logic behind CAI systems is mostly related to the TRIZ theory, i.e. to the refusal of trade-offs; thus, they are apparently in conflict with the logic of optimization, seen as minimization of negative issues within a given set of constraints. Nevertheless, as explained below, optimization systems can be used in a novel mode, such that they can play a relevant role in the identification of contradictions.

More specifically, the traditional approach to optimization involves the application of a complete system of constraints and loads to the geometry for describing all the design requirements.

It is worth to notice that this "optimal" i.e. "best compromise" solution is unnecessarily satisfying. It's often useful, before moving towards the detailed definition of the product architecture, to re-discuss already made assumptions, in order to obtain a solution which better satisfies general system objectives. On the basis of these considerations, the authors propose to perform a set of mono-objective optimization tasks in order to put in evidence conflicts among geometrical elements of the system under analysis.

Therefore, the work presented in this paper has been focalized on the definition of guidelines to make systematic the translation of a system functional model and its design requirements into a set of mono-objective optimization problems, aiming at satisfying a single requirement at one time. These guidelines have to be intended as a help for designer when they are asked to translate the functional architecture obtained as output of CAI system into a set of design variables and a set of constraints and load conditions to be optimized separately.

\subsection{From optimization analysis to geometrical contradictions identification}

The rationale behind the adoption of Optimization systems as a means for design analysis is the following:

- defining a single multi-goal optimization problem leads to a compromise solution;

- besides, defining $\mathrm{N}$ complementary mono-goal optimization problems, each with specific boundary conditions, leads to $\mathrm{N}$ different solutions;

- these solutions can be conflicting and this is the key to find contradictions.

According to this statement, the PROSIT design flow is structured as depicted in Fig. 2. The process starts with the definition of a set of single-goal optimization tasks, each representing a specific operating condition and/or a given design requirement. If each output solution satisfies the design objectives and they mutually fit each other, the process doesn't require any iteration and a detailed CAD model can be produced: the definition of a bridge between Optimization and PLM systems is a further goal of the PROSIT project, but it will be briefly presented below. 
Besides, if the solution of at least one of the optimization tasks doesn't fit the design requirements and/or the optimization tasks lead to conflicting geometries, the system must be further investigated in order to extract the geometrical contradictions. Geometrical contradiction concept is based onto studies published by Vikentiev about Geometrical Effects (GE). Somehow it can be stated that "GE start where physical and chemical effects end", or more precisely, unlike chemical effects, which enable to obtain some substances from others by the absorption or isolation of energy, and physical effects that enable to transform one form of energy into another, GE usually organize and redistribute flows of energy and substances that are already available in the system. In considered context, talking about design embodiment, functional architecture of system is already defined and then the work is essentially focused on geometrical field.

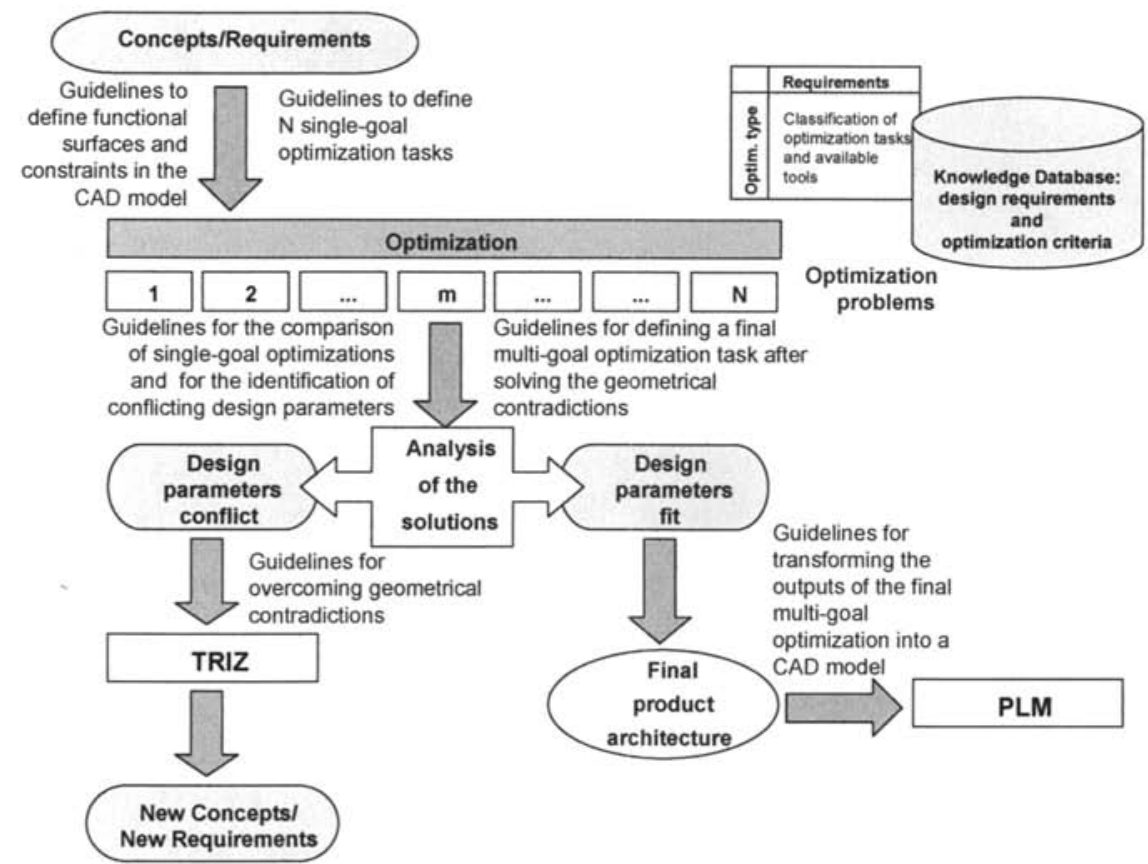

Fig. 2. Design flow according to the PROSIT approach

More specifically, according to the differences between the results of the singlegoal optimization tasks and to the nature of the conflicting design parameters, the geometrical contradictions can be classified into:

- 1D/2D/3D size contradictions: a linear dimension (e.g. length, width, height, thickness etc) should be high and should be small; a surface/volume should be big and should be small etc.

- shape contradictions: an element or a detail should assume different forms (e.g. sharp and rounded, circular and polygonal etc); 
- topological contradictions: an element or a detail should assume different topologies and/or orientations (e.g. monolithic and segmented, horizontal and vertical etc.).

A detailed description of the nature of these contradictions and the strategies to overcome them is out of the scopes of the present paper. Interested readers can find further details in [11].

Basically the methodology guides the designer according to the classical TRIZ approach. The application of TRIZ instruments allows, in synthesis to: identify the operational zone within the design space where the geometrical contradiction resides, check if contradictory requirements for the design parameters co-exist in the operational zone and time, check if those contradictory requirements co-exist under any condition and evaluate the opportunity to overcome them by means of a separation strategy or through a transition to subsystem elements or to its supersystem.

Closing the loop, as a result of this activity, a new set of optimization problems can be identified and can be solved making use of the optimization tools. In other words, the TRIZ principles are used to redefine the design volume, the functional surfaces and/or the optimization constraints so that the conflict between the design parameters disappears.

This procedure has to be iterated until optimization process' results converge, i.e. the geometries generated by the different single-goal optimization tasks fit each other. The final output of the Optimization system must be translated into a complete feature-based CAD model. It is worth to note that despite several features recognition systems are actually available on the market as additional modules of more advanced PLM systems, those algorithms typically fail, due to the intrinsic complexity of the shapes obtained in the previous step.

Thus, the proposed approach consists in the definition of a best practice implemented in a Knowledge Base user interface, which supports the designer in the translation of the output of a design optimization into a feature-based model, with simplified and possibly automated steps. Therefore, the project is oriented to capture the designer intent and knowledge during the optimization process through a KB interface capable to automate, support, and simplify geometric model rebuilding operations.

The development of the Knowledge Base relies on two main postulates:

- whatever is the mechanical part to be modeled, it is possible to segment the model into a set of invariants "typical" volumes, on the base of the product and design requirements;

- these "typical" volumes, characterized by a specific topology and some relations among geometric entities, can be adopted as seeds for the generation of the feature-based model after the optimization phase.

Further details about this work-package of the project will be published in the next future. 


\section{Study Cases}

The methodology here presented is currently under validation and it has been applied in several study cases in order to verify its feasibility, its benefits and drawbacks, in terms of time, quality and costs improvements, and in terms of capability to establish more collaborative working approaches. Five main fields of interest have been identified and they concern the re-design of the following mechanical systems:

- a scooter wheel using polyamide;

- a connecting rod for racing internal combustion engines;

- a hinge for glass doors;

- a hinge for chest freezers;

- a climbing hook.

Due to space limitations just the first design task will be detailed in order to clarify the PROSIT methodology with an exhaustive example.

\subsection{Re-design of a scooter wheel}

This test case has been inspired by a real case study developed during a collaboration of the authors with the Italian motorbike producer Piaggio [12]. The goal of the project was the design of a plastic wheel for light moto-scooters mainly aimed at costs reduction, of course without compromising safety and mechanical performances.

The traditional approach in Piaggio to assess the conformity of a wheel consists in three different experimental tests:

- deformation energy under high radial loads/displacements (simulating an impact against an obstacle);

- fatigue strength under rotary bending loads (simulating the operating conditions such as curves);

- fatigue strength under alternate torsional loads (simulating the accelerations and decelerations).

These tests have been adopted as reference criteria for design optimization, under the constraint of manufacturability through die-molding and the goal of minimizing mass since this parameter is directly related to costs.

Two functional surfaces were identified: the hub and the rim. The design domain for the topological optimization task coincides with the envelope of the volume of a classical wheel, while the internal side of the rim and the hub are assumed as invariant.

According to the proposed methodology, the first step consisted in the definition of three different mono-requirement topological optimizations related to the above mentioned complementary tests. As a result, two different mass distributions were generated, the first related to the radial and torsional tests, the second to the rotary bending test (Fig. 3): more specifically, the first geometry suggests the creation of a flat web between the hub and the rim; while the second leads to a number of radial spokes with transversal ribs. Before than facing such a topological contradiction, a 
further investigation must be done because the analysis revealed that the radial test by itself didn't meet the expected requirements.

When a mono-goal optimization doesn't converge to any acceptable solutions, the PROSIT guidelines suggest to split the problem further and to define a subset of optimization tasks with the same objective function, by removing the optimization constraints one-by-one.

As a result the user is driven to the identification of design constraints in the following form: the constraint $\mathrm{X}$ should not be respected in order to fulfill the goal $\mathrm{G}$, but it should be respected in order to satisfy the requirement $\mathrm{R}$.

In this case, the constraint to be removed in order to meet the design goal is the draw direction for manufacturability issues. In facts, the elimination of such a constraint leads to the geometry shown in Fig. 4: a hollow wheel with a double web supporting the side of the rim. Hence the contradiction can be expressed in the form: "the wheel should present an axial draw direction in order to preserve manufacturability and should not present an axial draw direction in order to provide a sufficient radial stiffness".
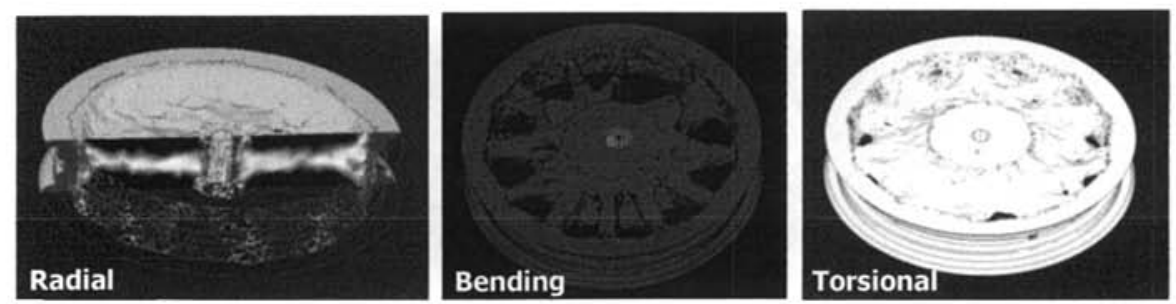

Fig. 3. Re-design of a scooter wheel: first step, comparison of the geometries arising from three complementary single-goal optimization tasks.
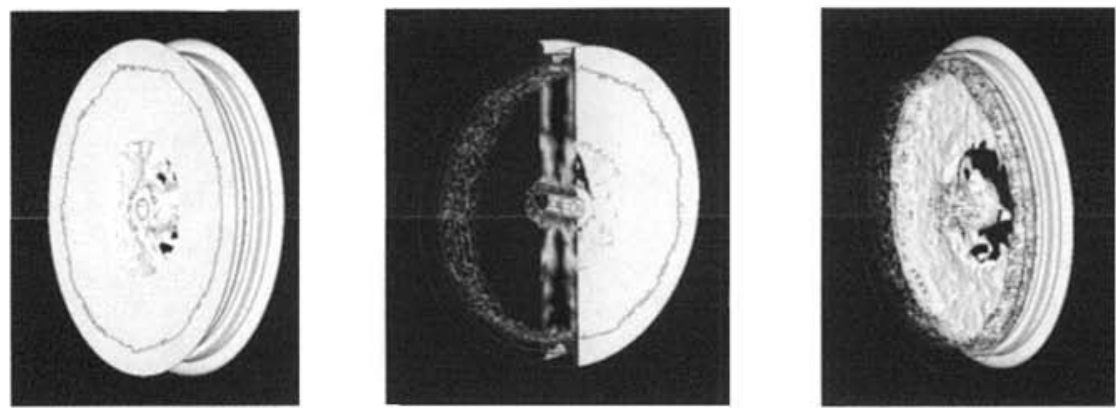

Fig. 4. Re-design of a scooter wheel: second step, in order to meet the design objectives, the manufacturability constraint should be removed. 

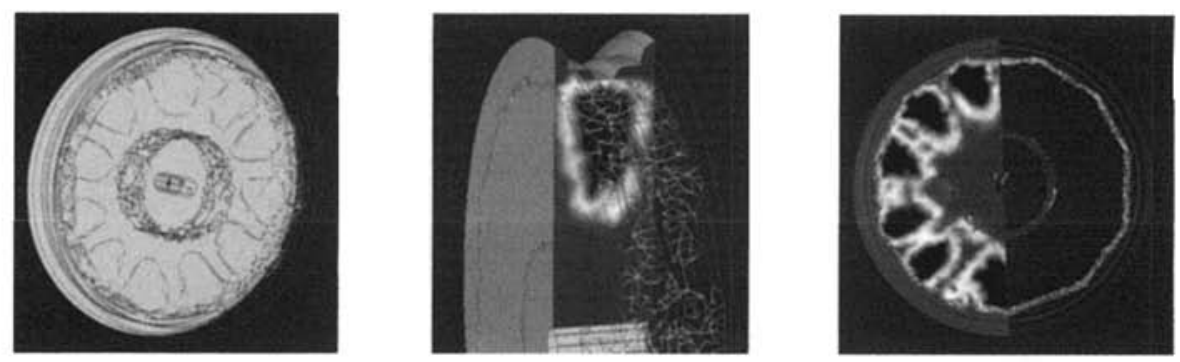

Fig. 5. Re-design of a scooter wheel: the geometrical contradiction can be overcome by applying the segmentation principle.

The guidelines extracted from the TRIZ instruments for overcoming the geometrical contradictions suggest, among the others, the application of the segmentation principle. In other words, the design space can be divided in two parts, so that the manufacturability is preserved when the two parts are separated, while the stiffness satisfies the requirements when assembled. Fig. 5 shows the results of the topological optimization obtained by dividing the wheel into two halves.

It is worth to note that the web is characterized by a number of transversal ribs that remind the spokes suggested by the optimization under rotary bending loads (Fig. 3). Therefore, in this case the solution of the contradiction within the radial load test brings to a geometry that fits with the results of the other mono-goal optimization tasks above defined and the overall process is converged.

This conceptual result can be translated into a geometrical model to be defined through technological features according to the second main goal of the PROSIT project. A standard FEM analysis of the final geometry revealed that a segmented plastic wheel made with a resin PA6 with a $30 \%$ volume content of glass fibers, (modulus of elasticity about $16 \mathrm{GPa}$ and stress to failure about $230 \mathrm{MPa}$ ) is capable to pass all the virtual tests required by Piaggio proprietary standards.

\section{Conclusions and Future Works}

The present paper has addressed the integration of Computer-Aided Innovation systems, Optimization systems and PLM/EKM tools as a means to improve the innovation resources and the efficiency of a product development cycle. The rationale of this research is the lack of formalized and validated procedures allowing the systematic introduction and integration of these tools in the design process. The development of these integration guidelines, that is the most innovative aspect of the research, should provide advantages in terms of design costs reduction, errors reduction, product quality improvement, process execution time and more effective internal and external knowledge use and share. 
A relevant aspect of the PROSIT project is the integration of apparently incompatible tools, thanks to the new role and way of usage of the Optimization Systems.

Moreover, in the mind of the authors, the methodology, through the definition of guidelines and best practices, can help overcoming classical interoperability problems affecting the design process, providing a common base for knowledge sharing and for a better interconnection between different systems and applications.

It is worth to notice that the PROSIT project doesn't aim at the creation of a fully automatic system for design embodiment, because both the comprehension of the root-cause of a geometrical contradiction and, most of all, the translation of the TRIZ principles into a new set of optimization tasks, requires a creative even if systematic step, demanded to the designer. Besides, the results obtained so far suggest the investigation of semi-automatic procedures to speed-up some routinary tasks like the comparison of the outputs of the single-goal optimization tasks as well as the removal of the optimization constraints one-by-one, when a single optimization doesn't converge to a solution.

\section{Acknowledgements}

The PROSIT project (Product development and systematic innovation www.kaemart.it/prosit) is co-funded by the Italian Ministry of University and Research.

The authors would like to thank M. Bordegoni e M. Bertoni from Politecnico di Milano and F. Rotini and F. Frillici from Università di Firenze, M. Muzzupappa from Università della Calabria and $\mathrm{F}$. Cappello from Università di Palermo for their contribution to the development of the PROSIT project.

\section{References}

1. G. Cascini: "State-of-the-Art and trends of Computer-Aided Innovation tools - Towards the integration within the Product Development Cycle", Building the Information Society, Kluwer Academic Publishers (ISBN 1-4020-8156-1), 2004, pp. 461-470.

2. M.P. Bendsoe, O. Sigmund: “Topology Optimization - Theory, Methods and Applications", Springer, 2003.

3. M. Bordegoni, U. Cugini: "Knowledge-driven method for integrated design", Proceedings of the International CIRP Design Seminar, Grenoble, France, May 12-14, 2003.

4. Simon Szykman, Steven J. Fenvesa, Walid Keirouzb, Steven B. Shooter: "A foundation for interoperability in next-generation product development systems", Computer-Aided Design, Volume 33, Issue 7, June 2001, Pages 545-559.

5. F. Mervyn, A. Senthil Kumar, S.H. Bok, A.Y.C Nee: "Developing distributed applications for integrated product and process design", Computer-Aided Design, Volume 36, Issue 8, July 2004, Pages 679-689. 
6. Barone S., Beghini M., Bertini L. "Coupled Structure Analysis and Geometric Modelling in Rule-based Shape Optimisation". Proc. of The 11th ADM International Conference on Design Tools And Methods in Industrial Engineering, Vol. A., pp. 169-176, 1999

7. Rosen D.W., Grosse I.R., "A feature based shape optimization technique for the configuration and parametric design of flat plates". Engineering with Computers, Vol. 8, pp. 81-91, 1992

8. Takezawa A. et al. "Structural optimization for concurrent design and analysis at the conceptual design stage". In Advanced Design, Production and Management Systems, A.A. Balkema Publishers, pp. 9-16, 2003

9. Spath D., Neithardt W., Bangert C. "Integration of Topology and Shape Optimization in the Design Process". Proc. of the 2001 International CIRP Design Seminar, 2001

10. Bordegoni M., Cascini G., Filippi U. and Mandorli F., "A methodology for evaluating the adoption of knowledge and innovation management tools in a product development process", ASME Design Technical Conferences, Chicago September 2-6 2003.

11. G. Cascini, P. Rissone, F. Rotini: "From design optimization systems to geometrical contradictions", accepted for publication into the Proceedings of the 7th ETRIA TRIZ Future Conference, Frankfurt, Germany, 6-8 November 2007.

12. Cascini G., Rissone P.: "Plastics design: integrating TRIZ creativity and semantic knowledge portals", Journal of Engineering Design, vol. 15, no. 4, August 2004, Special Issue: "Knowledge Engineering \& Management Issues in Engineering Design Practices", pp. 405-424. 